\title{
Outcome and Quality of Life in Patients Treated for Abdominal Aortic Aneurysms: A Single Center Experience
}

\author{
Florian Dick - Véronique Grobéty · Franz F. Immer · Dai Do Do · \\ Hannu Savolainen · Thierry P. Carrel · Jürg Schmidli
}

Published online: 29 March 2008

(c) Société Internationale de Chirurgie 2008

\begin{abstract}
Background Durability of protection and long-term quality of life (QoL) are critical outcome parameters of abdominal aortic aneurysm (AAA) repair. The aim of the present study was to compare results of endovascular and open aneurysm repair (EVAR and OR) with adjusted standard populations, including stratification for urgency of presentation.

Methods Retrospective analysis of prospectively collected data of 401 consecutive patients presenting with AAA between January 1998 and December 2002. Crosssectional follow up was $58 \pm 29$ months. Patients were grouped into three cohorts: elective $\operatorname{EVAR}(n=68)$, elective OR $(n=244)$, and emergency OR (including symptomatic and ruptured AAA, $n=89$ ). Endpoints were perioperative (i.e., 30 days or in-hospital) and late mortality rates, as well as long-term QoL as assessed by the Short Form health survey questionnaire (SF-36).

Results Mean age was lower in the elective OR cohort (66 \pm 10 years) than in the EVAR cohort ( $72 \pm 7$ years; $p<.05)$. Perioperative mortality rates were $4.4 \%, 0.4 \%$, and $10.1 \%$, for the EVAR, elective OR, and emergency OR cohorts, respectively $(p<.05)$. Corresponding cumulative survival rates after 4 years were $67 \%, 89 \%$, and $69 \%$, respectively. Long-term QoL SF-36 scores were in all cohorts similar to age- and gender-adjusted standard
\end{abstract}

F. Dick · V. Grobéty · F. F. Immer $(\bowtie) \cdot$ H. Savolainen ·

T. P. Carrel · J. Schmidli

Department of Cardiovascular Surgery, Inselspital, University

Hospital Bern, and University of Bern, Berne 3010, Switzerland

e-mail: franzimmer@yahoo.de

D. D. Do

Division of Clinical and Interventional Angiology, Swiss Cardiovascular Center, Inselspital, University Hospital Bern, and University of Bern, Bern 3010, Switzerland populations, which score between 85 and 115: $99.6 \pm 35.8$ (EVAR), $101.3 \pm 32.4$ (elective OR), and $100.4 \pm 36.5$ (emergency OR).

Conclusions Long-term QoL is not permanently impaired after AAA repair, but returns in long-term survivors to what would be expected in a standard population. In this respect, differences were found neither between EVAR and OR, nor between elective and emergency repair. Perioperative mortality rates were highest in patients undergoing emergency OR. The outlook for such patients after the perioperative period, however, was similar to that for patients undergoing elective repair.

\section{Introduction}

Repair of abdominal aortic aneurysms (AAA) can be achieved by either open or endovascular aneurysm repair (OR or EVAR). Both treatment modalities are well established in terms of perioperative morbidity and mortality, long-term survival, and cost-effectiveness [1-7], and both feature distinct advantages and disadvantages. Specifically, EVAR is less invasive than OR and is associated with significantly lower operative mortality $[8,9]$. However, its long-term durability is still uncertain, and serious concerns have been raised because long-term complications have been reported in 25-40\% of patients. Many of the patients with such complications may need additional interventions or-uncommonly-even conversion to open surgery during follow-up. Moreover, the ultimate AAA complication-i.e., continuing aneurysm expansion and rupture-cannot be prevented once and for all. Therefore, close surveillance of EVAR patients over many years is still considered mandatory $[5,6,10-14]$. 
Health related quality of life (QoL) is increasingly recognized as a critical parameter in the assessment of outcome [15]. Although a faster return to normal QoL has been ascribed to EVAR, long-term QoL was found to be significantly higher after OR after the first 6 months [13]. The patient's uncertainty about long-term durability of EVAR, as made clear to the individual by close endograft surveillance, might underlie this difference. Emergency repair of ruptured AAA ( $\mathrm{AAAA}$ ) on the other hand, while associated with relevant operative mortality, has been reported to barely affect long-term QoL [16]. Such data are essential to justify high attendant financial costs of AAA repair [17, 18] and to determine resource allocation to different treatment options [4].

The aim of this single-center series was to analyze longterm survival as well as long-term QoL of surviving patients after EVAR and OR, respectively, and to compare them with an age- and gender-adjusted standard population. Stratification for urgency of presentation (i.e., elective versus emergency repair) was included.

\section{Materials and methods}

Post hoc analysis was carried out on a prospectively registered, consecutive series of 401 patients who were treated for AAA between January 1998 and December 2002 at our institution. Mean age of patients was $68 \pm 9$ years, and 349 patients were men (87\%). Collected information included pre-existent co-morbidities, cardiovascular risk factors, aneurysm morphology, as well as in-hospital morbidity, mortality, and length of stay (Table 1). Identification of risk factors was based on previously published definitions [19]. If percutaneous

Table 1 Demographic characteristics, preoperative data, and in-hospital outcome

\begin{tabular}{|c|c|c|c|c|c|c|}
\hline & EVAR & & Elective OAR & & Emergency OAR & \\
\hline \multicolumn{7}{|l|}{ Demographics } \\
\hline Number of patients & 68 & $17.0 \%$ & 244 & $60.8 \%$ & 89 & $22.2 \%$ \\
\hline Male gender & 64 & $94.1 \%$ & 203 & $83.2 \%$ & 82 & $92.1 \%$ \\
\hline Age, years & $71.7 \pm 7.2$ & & $66.4 \pm 10.0^{*}$ & & $70.6 \pm 9.1$ & \\
\hline \multicolumn{7}{|l|}{ Preoperative data } \\
\hline Diameter, cm & $5.9 \pm 1.2$ & & $6.8 \pm 1.4^{*}$ & & $7.5 \pm 1.8^{*}$ & \\
\hline Diabetes & 6 & $8.8 \%$ & 35 & $14.3 \%$ & 5 & $5.6 \%$ \\
\hline Tobacco use & 30 & $44.1 \%$ & 94 & $38.5 \%$ & 39 & $43.8 \%$ \\
\hline Hypertension & 37 & $54.4 \%$ & 111 & $45.5 \%$ & 52 & $58.4 \%$ \\
\hline Hyperlipidemia & 18 & $26.5 \%$ & 79 & $32.4 \%$ & 16 & $18.0 \%$ \\
\hline Coronary artery disease & 28 & $41.2 \%$ & 74 & $30.3 \%$ & 34 & $38.2 \%$ \\
\hline History of MI & 18 & $26.5 \%$ & 56 & $23.0 \%$ & 24 & $27.0 \%$ \\
\hline PTCA & 11 & $16.2 \%$ & 19 & $7.8 \%$ & 11 & $12.4 \%$ \\
\hline CABG & 6 & $8.8 \%$ & 11 & $4.5 \%$ & 3 & $3.4 \%$ \\
\hline COPD & 15 & $22.1 \%$ & $18^{*}$ & $47.4 \%$ & 22 & $24.7 \%$ \\
\hline \multicolumn{7}{|l|}{ In-hospital outcome } \\
\hline Mortality & 3 & $4.4 \%$ & 1 & $0.4 \%$ & $9^{*}$ & $10.1 \%$ \\
\hline Bleeding & 0 & $0.0 \%$ & 6 & $2.5 \%$ & 5 & $5.6 \%$ \\
\hline Infection & 2 & $2.9 \%$ & 14 & $5.7 \%$ & 5 & $5.6 \%$ \\
\hline Limb ischemia & 2 & $2.9 \%$ & 8 & $3.3 \%$ & 5 & $5.6 \%$ \\
\hline Immediate conversion & 1 & $1.5 \%$ & 0 & $0.0 \%$ & 0 & $0.0 \%$ \\
\hline \multicolumn{7}{|l|}{ Length of stay } \\
\hline Intensive care, days & $0.3 \pm 0.3$ & & $0.8 \pm 0.5$ & & $1.8 \pm 2.1^{*}$ & \\
\hline Overall, days & $8.3 \pm 7.6$ & & $10.2 \pm 7.2 *$ & & $14.8 \pm 8.6^{*}$ & \\
\hline
\end{tabular}

In-hospital data for patients who underwent repair of abdominal aortic aneurysm (AAA) either by endovascular aortic repair (EVAR: $n=68$ ), elective open aortic repair (OR: $n=244$ ); or emergency OR (i.e., symptomatic or ruptured AAA: $n=89$ ). EVAR and elective OR patients were postoperatively monitored on an intermediate care unit ( $2.3 \pm 2.4$ and $2.8 \pm 1.2$ days, respectively). Results are displayed as absolute values or as mean values $\pm \mathrm{SD}$

$M$, myocardial infarction; $P T C A$, percutaneous transluminal coronary angioplasty; $C A B G$, coronary artery bypass grafting; $C O P D$, chronic obstructive pulmonary disease

$* p<0.05$ comparing EVAR to OAR (elective or emergency) 
transluminal coronary angioplasty (PTCA) had recently been performed, scheduled aortic repair was postponed for 8-12 weeks. Approval of the local ethics committee was obtained before outcome analysis.

Aortic abdominal aneurysms were treated (1) if they presented with a maximum transverse infrarenal aortic diameter of $50-55 \mathrm{~mm}$ or more, (2) if imaging evidence documented AAA of 1.5 times the reference aortic diameter with an expansion rate of $>10 \%$ per year, or (3) if they were symptomatic or had ruptured. During the study period, indications for EVAR were limited to patients over 65 years of age with a high surgical risk and with favorable aneurysm morphology, i.e., a long infrarenal aortic neck (i.e., longer than or equal to $20 \mathrm{~mm}$ without conical shape), absence of mural thrombus at the level of the aortic neck, an aortic neck angulation of less than $60^{\circ}$ with respect to the aneurysm axis, as well as non-tortuous and patent pelvic axes on both sides. Additionally, at least one hypogastric artery had to be deemed preservable. Appreciation of urgency was based on history, presenting symptoms, and computed tomographic angiographies, which were obtained whenever patients were stable enough preoperatively. Rupture of AAA was defined as loss of continuity of the aortic wall in the region of aneurysm associated with fresh blood outside the wall of the aneurysm. In order to be classified in this way, rupture had to be confirmed by intraoperative finding of a retroperitoneal hematoma or suffusion and a rupture site in the aneurysm. All other patients with clinical suspicion of rupture or exquisitely painful aneurysms on direct palpation were considered symptomatic, but still urgent. Individual decisions regarding treatment of AAA were left to the discretion of the attending surgeon with consideration of patient request.

All patients were followed systematically after aortic repair in a specialized outpatient clinic: after OR, patients were seen after 3 months, and annually to every second year thereafter for an abdominal duplex sonography check. After EVAR, patients returned after 3, 6, 12, 18, and 24 months and annually thereafter as proposed by the EUROSTAR protocol [12]. Additionally, from October 2000 on, all eligible EVAR patients were included in the EUROSTAR registry.

For cross-sectional assessment of present-day QoL, all surviving patients were contacted between January and June 2006 by means of the validated German version of the self-administered Short Form health survey questionnaire (SF-36). The questionnaire was sent a second time to nonresponders before they were contacted by phone. Mean follow-up constituted $58 \pm 29$ months. Outcome was analyzed according type of aortic repair, i.e., elective OR, emergency OR, and EVAR.
Study endpoints

Perioperative mortality and morbidity included all events within 30 days of treatment or when the patient was still in the hospital. Collected events included surgical site infections and severe bleeds, as well as occurrence of acute lower limb ischemia, and were registered if they prompted surgical revision during the same hospital stay. Cumulative longterm survival was assessed according to the method proposed by Kaplan and Meier [20]. Quality of life was assessed with the self-administered SF-36 questionnaire [21-23]. Details of this validated questionnaire have been published previously [24]. Briefly, the SF-36 consists of 36 short questions reflecting QoL in eight different aspects: bodily pain (abbreviated BP, two items); mental health (MH, five items); vitality (VT, four items); social functioning (SF, two items); general health ( $\mathrm{GH}$, five items); physical functioning ( $\mathrm{PF}, 10$ items); and role functioning, both emotional (RE, three items) and physical (RP, four items). Role functioning reflects the impact of emotional and physical disability on work and regular activity (the individual's normal everyday role). Raw points generate a score for each dimension, which add up to a total raw score ranging from 0 to 100 , with 100 reflecting best functioning. Raw scores then were adjusted for age and gender by multiplication with the appropriate factor based on a validated Western European standard population (Sweden, $n=8,930$ ) according to the SF-36 manual [22, 23], thereby generating an adjusted score. Normal values for the adjusted score range from 85 to 115 .

\section{Statistical analysis}

Continuous variables are summarized as mean $( \pm$ one standard deviation $[\mathrm{SD}]$ ), when normally distributed, and as median (range), when asymmetrically distributed. Categorical variables are presented as numbers (percentages). Differences between continuous variables were compared by two-tailed unpaired $t$-test if normally distributed, and by the Mann-Whitney $U$-test, if asymmetrically distributed. Categorical variables were compared by two-tailed Fisher's exact test. Kaplan-Meier survival estimates were correlated by $\chi^{2}$ testing with estimated actual mortality risks of the age-adjusted Swiss population as published by the Swiss Federal Statistical Office (http://www.bfs. admin.ch/bfs/portal/en/index.html). SF-36 scores were analyzed in accordance with the SF-36 manual, replacing missing values using the described algorithm [22, 23]. After adjustment for age and gender, SF-36 scores were compared by means of Mann-Whitney $U$-test. Results were stratified for modality of treatment and urgency of presentation. A $p$-value of less than 0.05 was considered to indicate statistical significance. All analyses were 
performed using a computerized software package, SPSS for Windows, version 15.0.

\section{Results}

Of the 401 patients with AAA, 244 underwent elective OR (60.1\%), 89 were treated by emergency OR $(22.2 \%)$, and 68 patients received elective EVAR (17.0\%). Of the urgent patients, 51 presented with rAAA (57.3\%). Differences between treatment cohorts regarding demographic and preoperative characteristics, as well as in-hospital outcome are given in Table 1. Patients treated by elective OR were significantly younger than EVAR patients $(66 \pm 10$ years versus $72 \pm 7$ years; $p<0.05)$. The EVAR patients, however, presented with significantly smaller AAA-diameters than patients treated by either elective or emergency OR $(p<0.05)$. Distribution of cardiovascular risk factors was similar in all treatment cohorts $(p=n s)$. However, history of previous myocardial infarction, PTCA and coronary artery bypass graft (CABG)-surgery, as well as presence of chronic obstructive pulmonary disease (COPD), were both significantly more prevalent in patients who underwent EVAR. One of the elective OR patients had had EVAR prior to actual aortic repair.

Thirteen patients died while still in hospital, giving an overall perioperative mortality rate of $3.2 \%$. Stratified rates were $4.4,0.4$, and $10.1 \%$ for the EVAR, elective OR, and emergency OR cohorts, respectively $(p<0.05)$. Length of hospital stay was significantly longer in patients who underwent emergency OR (14.8 \pm 8.6 days), as compared to elective OR or EVAR $(10.2 \pm 7.2$ and $8.3 \pm 7.6$ days, respectively; $p<0.05$ ). Of 388 discharged patients, 61 died during follow-up (15.7\%; Table 2), accounting for cumulative survival estimates of 86 (EVAR), 97 (elective OR), and $85 \%$ (emergency OR) at 1 year, and 67, 89, and $69 \%$ at 4 years, respectively (Fig. 1). Based on the sample size of the cohorts, no statistically significant differences were found when compared to the expected 4-year survival rate of the Swiss population, which actually is estimated to

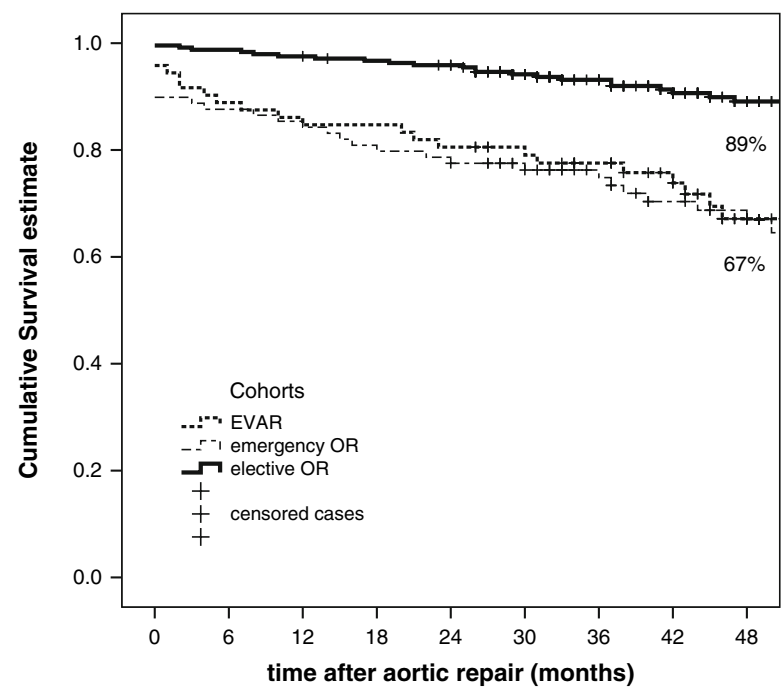

\begin{tabular}{lccccc}
\hline EVAR & 65 & 58 & 54 & 43 & 22 \\
Eled. OAR & 243 & 238 & 231 & 165 & 106 \\
Emerg. OAR & 80 & 76 & 70 & 53 & 40
\end{tabular}

Fig. 1 Cumulative survival rates after abdominal aortic aneurysm (AAA) repair as assessed by the Kaplan-Meier method and stratified for treatment cohorts. EVAR endovascular aneurysm repair; OR open aneurysm repair. Emergency OR included repair of symptomatic and ruptured AAA

be around $90 \%$ for 66 -year-olds, and around $85 \%$ for 71 year-olds.

All survivors ( $n=327)$ could be contacted for follow-up (100\%). Forty-three patients, however, did not answer the questionnaire, either due to language problems, medical reasons (end-stage tumor, cerebrovascular incident, $n=6$ ), or refusal. However, all non-responders were contacted by phone and all were alive. Overall return rate of questionnaires among surviving patients therefore was $86.8 \%$.

Long-term QoL (measured after $58 \pm 29$ months) was good in all treatment cohorts, with an average SF-36 score of $99.6 \pm 35.8$ in the EVAR cohort, $101.2 \pm 35.2$ in the elective OR cohort, and $100.4 \pm 36.5$ in the emergency OR cohort. These results are similar to data obtained in an age-

Table 2 Perioperative and long-term mortality

\begin{tabular}{|c|c|c|c|c|c|c|}
\hline & EVAR & & Elective OAR & & Emergency OAR & \\
\hline \multicolumn{7}{|l|}{ Early (30 day) } \\
\hline Patients $n(\%)$ & 68 & $17.0 \%$ & 244 & $60.8 \%$ & 89 & $22.2 \%$ \\
\hline Perioperative mortality $n(\%)$ & 3 & $4.4 \%$ & 1 & $0.4 \%$ & 9 & $10.1 \%$ \\
\hline \multicolumn{7}{|l|}{ Follow-up } \\
\hline Average, years & $4.6 \pm 1.9$ & & $4.9 \pm 2.5$ & & $4.8 \pm 2.0$ & \\
\hline Crude mortality $n(\%)$ & 19 & $27.9 \%$ & 22 & $9.0 \%$ & 20 & $22.5 \%$ \\
\hline
\end{tabular}

Follow-up data for patients who underwent repair of AAA either by EVAR $(n=68)$, OR $(n=244)$; or emergency OR (i.e., symptomatic or ruptured AAA: $n=89$ ). Perioperative mortality includes all deaths occurring within first 30 days after procedure, and of all patients continuously hospitalized until death 
and gender-matched standard population, which scores between 85 and 115. Separate analysis of individual physical and psychological domains showed the same results: All three cohorts scored within normal range in both physical and mental domains, after adjustment for age and gender. Particularly in the key dimension mental health, all cohorts scored in the same range, rendering the cohorts actually comparable for all other domains of the SF-36.

\section{Discussion}

Being the thirteenth leading cause of death in the United States, AAA is an important and costly health concern. Although OR basically has prevailed since its introduction on 29 March 1951 [25], it still carries substantial morbidity and mortality. The most important reasons are the extensive operative trauma, the changes in renal blood flow induced by aortic clamping, the blood loss, and the ischemia-reperfusion injury to the lower part of the body and the intestines. Hence, OR is currently being challenged by minimally invasive techniques (EVAR) as the treatment of choice for AAA. Elective operative mortality and morbidity have been established quite congruently for both modalities, with reported results depending on the respective study design. Accepted 30-day mortality rates range around $3.8 \%$ for elective OR [7], and around $1.5 \%$ for elective EVAR [8, 9]. However, EVAR offered no midterm advantage over OR with respect to all-cause mortality and QoL in a randomized controlled trial (RCT) [5], but it was more expensive and led to a greater number of complications and additional interventions. Thus, the long-term efficacy and durability of EVAR remains uncertain, since its perioperative advantages might be lost after the first postoperative year [6]. Reports on corresponding rates for emergency repair of AAA are less congruent, in part because discrimination between symptomatic and rAAA is not consistently reported, and in part because overall mortality versus in-hospital mortality cannot always be differentiated. Reported results therefore range from $15 \%$ to $90 \%$ but are usually quoted approximately as $40-45 \%$ for emergency OR [17, 26].

Assessing the outcome of surgery simply by crude morbidity and mortality rates fails to take into account the patient's perspective. Quality of Life essentially subsumes the relative importance of all intervention-related sequelae to the results noted by the patient, and its assessment has therefore become increasingly important in many fields of medicine; likewise in vascular surgery [15]. A number of studies have assessed postoperative QoL after elective or emergency AAA repair [4, 16, 27-32], and some have even compared QoL after OR and EVAR in a RCT design $[5,13]$. As expected, patients treated by EVAR invariably returned earlier than OR patients to preoperative QoL. Surprisingly, however, OR patients had a significantly better QoL than EVAR patients from 6 months on in one RCT [13]. Contrary to common suggestions in the literature [29, 30], the authors of the DREAM trial did not ascribe this relative QoL impairment to a close surveillance program after EVAR, because both study arms were monitored equally. They rather suggested that knowledge of having definitely survived a severe illness and major surgery plays a critical role. In line with that conclusion, long-term QoL after emergency OR has been found to be equal to elective OR, as well as to age- and gender-adjusted controls [4, 16]. However, in another series the radical change experienced by patients who underwent an emergency OR was followed by deterioration in QoL, whereas those who had elective OR reported improved QoL, which relativizes this notion [27].

In our series 401 consecutive patients with large or symptomatic AAA were included over a period of 5 years. The study population therefore reflects the real life spectrum of AAA pathology in a tertiary referral center. There was no loss to follow up. For cross-sectional QoL assessment among survivors, a validated tool was used (SF-36) with a representative return rate $(86.8 \%)$. Most of the other mentioned studies concentrating on QoL after AAA repair were found to investigate either small or selected samples, to be retrospective with incomplete follow-up, or not to have used validated QoL assessment tools in a systematic review [4]. The QoL assessment instruments used, as described in this review were heterogeneous and ranged from non-validated, disease-specific, and self-designed questionnaires to generic and complex multidimensional interrogation tools. Direct comparisons of results may therefore be extremely delicate. The main advantage of the SF-36 QoL questionnaire as used in the present study is its reliable validation and broad acceptance. The generic design renders it widely applicable, and its methodology has been used successfully for vascular patients before by our group [24, 33] and others [4, 15]. Intriguingly, statistical adjustments for age and gender allow comparisons with standardized Western European control populations [22, 23]. SF-36 thereby presumably conveys a reasonable notion of the range a normal QoL could be expected to be for the respective demographic segment.

The main finding of our study was that long-term QoL returned to a normal range independent of treatment modality or urgency. Hence, QoL was equal to age- and gender-matched healthy subjects 4-5 years after aortic repair in all treatment cohorts. This is in line with was has been found in a recent systematic review for emergency AAA repair, although all included studies had smaller sample sizes than our emergency OR cohort [4]. For elective AAA repair, however, our series did not reproduce 
the findings of the DREAM trial, where OR patients had a relatively better postoperative QoL in the long term than EVAR patients [13]. Indeed, no statistically significant differences were found between elective OR and EVAR patients regarding long-term QoL in our series. As all treatment cohorts returned to the same QoL as their healthy peers, the interpretation that aneurysm repair did not deteriorate QoL in these risk populations is probably justified and seems to be particularly true for EVAR patients, who represented the least favorable patient selection. The study thereby certainly corroborates the findings of the EVAR 1 trial in this respect [5]. However, it must be borne in mind that only long-term survivors were assessed, and they could be suspected to represent favorable selection, not only with respect to co-morbidities but also to QoL.

In comparison to the literature, mortality rates were very low in the OR cohorts. In particular, emergency OR patients had a perioperative mortality rate $(10.1 \%)$ that lies well beneath commonly published rates (15-90\%) [17]. The First Vascunet Database Report 2007 of the European Society for Vascular Surgery (ESVS) describes outcome of over 33,000 AAA patients in six Western countries [26]. In these pooled data, operative mortality in the corresponding age group was around $35 \%$ for emergency OR. The operative mortality for symptomatic AAA alone-i.e., excluding rAAA—was still $9.6 \%$. Therefore the assumption that our low operative mortality figure for emergency OR was simply based on the inclusion of symptomatic patients does not seem justified. A possible explanation for this favorable figure, however, is an extremely wellrehearsed team approach to symptomatic or rAAA. Therefore, the concept of permissive hypotension with hypotensive hemostasis, as propagated by Crawford and others since the early 1990s [34], was integrated into our rescue chain and includes paramedics, anesthesiologists, and vascular surgeons, who detain fluid resuscitation whenever possible until surgical control of hemorrhage has been reached.

Other important factors include preoperative imaging whenever possible, induction of general anesthesia and intubation only in the operating theatre, with the surgeon ready to cut, and the presence of at least two vascular surgeons supported by one or two assistants at such operations. A dense health care system in Switzerland, with short rescue paths and concentration of demanding vascular surgery in dedicated specialized centers further facilitates low operative mortality figures.

The fraction of emergency AAA (22\%) - and even more of rAAA (13\%) - is comparatively low in the presented material. Similar figures were found for the whole country in the First Vascunet Database Report 2007, where Switzerland had the lowest rate of emergency AAA repair $(<20 \%)$ as compared for instance to Denmark where this rate approached $50 \%$ (37\% for rAAA). It is unclear whether this difference is due to reporting bias or whether it reflects an improved aneurysm screening and surveillance program. During the study period, ten patients with rAAA were not operated on because of ongoing mechanical reanimation at admission. Unfortunately, we have no information on how many patients with rAAA were actually not referred to us or who died on the way during the same period of time.

With a similar team approach, operative mortality of elective OR can be kept comparably low in dedicated centers. In the present series, the figure was well below $1 \%$, whereas the Vascunet Database [26] reported an average mortality ten times higher for the same age group, i.e., around $5 \%$. The favorable impact of experience in patient management and high surgical volume has been endorsed in a recent survey of 131 German hospitals [35]. Operative mortality of elective EVAR, however, was somewhat elevated in the present series $(4.4 \%)$ when compared to recent literature, where figures around $2 \%$ are typically reported $[8,26]$. Several reasons may account for this finding. Our patient selection for EVAR shows a high prevalence of relevant cardiopulmonary co-morbidities (Table 1), which reflects the policy of our department at the time to mainly treat AAA patients with high surgical risk with EVAR. Furthermore, only patients of advanced age were offered EVAR. Another important factor may be that EVAR was not employed on a regular basis in our department until 1997. Therefore part of the learning curve might be incorporated into the analysis. Finally, improvements in device design since the end of the present study have led to drastic improvements in reported outcome.

Long-term survival after the perioperative period was similar to the expected survival of the age-matched Swiss population as estimated by the Swiss Federal Statistical Office for all treatment cohorts. However, whereas both OR cohorts displayed a fairly parallel trend of survival curves over 4 years, survival after EVAR fell to a somewhat steeper degree. Although, because of the small sample size, no statistically significant difference was found in comparison to a normal survival curve, the difference in survival after the different procedures probably reflects selection of patients with significant disease.

\section{Limitations of study}

Several limitations of this cross-sectional outcome analysis of prospectively collected treatment cohorts have to be addressed. Mean follow-up was almost 5 years, and it included a considerable variance, as expected of a study period of 5 years. Additionally, because this was a nonrandomized, observational series, basic requirements for comparability of treatment cohorts were not met (Table 1). 
Statistical control of results was sought by $z$-testing against the norms of standard populations. With regard to life expectancy, estimated Swiss norms could be used. As for QoL assessment, unfortunately no validated "Swiss norm" exists. The large Swedish series $(n=8,930)$ used for QoL comparison is a validated SF-36 reference population of people from similar socioeconomic backgrounds. Therefore a comparable QoL can probably safely be assumed. Furthermore, an ideal QoL assessment tool should be independent of geographic, linguistic, or cultural boundaries [15]. The SF-36-although certainly not meeting all criteria of the ideal-is a generic QoL instrument with a high degree of validation and acceptance. It thus has been recommended for use in vascular disease-related $\mathrm{QoL}$ assessments and outcome analyses, particularly because of its validated translations into many major languages, which make it a truly international scale of health-related QoL [15]. However, it certainly has its limitations, mainly affecting its sensitivity regarding bodily pain and functional impairments. Additionally, floor effects in role functioning have been described in very ill patients, where the test loses some of its discriminatory force for deterioration [15]. Combining it with disease-specific QoL instruments would certainly be desirable to compensate for some of these limitations. However, existing tools specific for vascular diseases, such as the VascuQoL, Walking Impairment Questionnaire, or PAD Questionnaire, all concentrate on peripheral arterial occlusive disease and therefore do not serve our purpose [36]. To our knowledge, at the time of this study, there was no validated QoL assessment tool specific for AAA patients.

As discussed above, a limitation of the cross-sectional QoL assessment is the lack of longitudinal information. Hence patients with favorable outcome may have been selected by this process, as no QoL information on patients who died during follow-up was available. Results therefore represent QoL of long-term survivors rather than QoL outcome of all originally treated patients. However, from the patients' perspective, the information that normal longterm QoL can be expected in survivors may be comforting. Whether the favorable long-term QoL in surviving patients is due solely to "natural selection" or whether, to a degree, it represents a limitation of SF-36 design is hard to determine.

Although the return rate of SF-36 questionnaires mailed to our patients was representative in all cohorts $(>80 \%)$, the missing information was probably not random. Whereas very few patients did not answer because of severe illness $(n=6)$, language problems accounted for the majority of the deficit. The primary language of the majority of our patients is German. We therefore used the validated German version of the SF-36. Hence the majority of missing questionnaires was due to the inability of many
Italian- or French-speaking patients to answer such a questionnaire in a foreign language. However, we have previously shown that patient characteristics basically are not different between the different linguistic parts of Switzerland [24].

In conclusion, long-term QoL is not permanently impaired after AAA repair. In long-term survivors it returns to what would be expected in an age- and genderadjusted standard population in all eight aspects assessed by the SF-36. In this respect, no differences could be found either between EVAR and OR or between elective and emergency repair. Perioperative mortality rates were highest in patients undergoing emergency OR. Their outlook after the perioperative period, however, is similar to that for patients undergoing elective repair.

Acknowledgments We thank our biostatistician, Brigitta Gahl, $\mathrm{MSc}$, for invaluable help with the analyses. Florian Dick was granted stipendiary support from the Swiss National Funds (SNF, PBBSB120858) and the Margarete und Walter Lichtenstein Stiftung, Basel, for an independent vascular research fellowship during part of his time.

\section{References}

1. Dardik A, Lin JW, Gordon TA et al (1999) Results of elective abdominal aortic aneurysm repair in the 1990s: a populationbased analysis of 2335 cases. J Vasc Surg 30:985-995

2. Hertzer NR, Mascha EJ, Karafa MT et al (2002) Open infrarenal abdominal aortic aneurysm repair: the Cleveland Clinic experience from 1989 to 1998. J Vasc Surg 35:1145-1154

3. Mangione CM, Goldman L, Orav EJ et al (1997) Health-related quality of life after elective surgery: measurement of longitudinal changes. J Gen Intern Med 12:686-697

4. Tambyraja AL, Fraser SC, Murie JA et al (2004) Quality of life after repair of ruptured abdominal aortic aneurysm. Eur J Vasc Endovasc Surg 28:229-233

5. Greenhalgh RM, Brown LC, Epstein D et al (2005) Endovascular aneurysm repair versus open repair in patients with abdominal aortic aneurysm (EVAR trial 1): randomised controlled trial. Lancet 365:2179-2186

6. Blankensteijn JD, de Jong SE, Prinssen M et al (2005) Two-year outcomes after conventional or endovascular repair of abdominal aortic aneurysms. N Engl J Med 352:2398-2405

7. Blankensteijn JD, Lindenburg FP, Van der Graaf Y et al (1998) Influence of study design on reported mortality and morbidity rates after abdominal aortic aneurysm repair. Br J Surg 85:1624-1630

8. Greenhalgh RM, Brown LC, Kwong GP et al (2004) Comparison of endovascular aneurysm repair with open repair in patients with abdominal aortic aneurysm (EVAR trial 1), 30-day operative mortality results: randomised controlled trial. Lancet 364:843-848

9. Prinssen M, Verhoeven EL, Buth J et al (2004) A randomized trial comparing conventional and endovascular repair of abdominal aortic aneurysms. N Engl J Med 351:1607-1618

10. Brewster DC (2002) Do current results of endovascular abdominal aortic aneurysm repair justify more widespread use? Surgery 131:363-367

11. Holzenbein TJ, Kretschmer G, Thurnher S et al (2001) Midterm durability of abdominal aortic aneurysm endograft repair: a word of caution. J Vasc Surg 33:S46-S54 
12. Laheij RJ, Buth J, Harris PL et al (2000) Need for secondary interventions after endovascular repair of abdominal aortic aneurysms. Intermediate-term follow-up results of a European collaborative registry (EUROSTAR). Br J Surg 87:1666-1673

13. Prinssen M, Buskens E, Blankensteijn JD (2004) Quality of life endovascular and open AAA repair. Results of a randomised trial. Eur J Vasc Endovasc Surg 27:121-127

14. van Marrewijk C, Buth J, Harris PL et al (2002) Significance of endoleaks after endovascular repair of abdominal aortic aneurysms: The EUROSTAR experience. J Vasc Surg 35:461473

15. Beattie DK, Golledge J, Greenhalgh RM et al (1997) Quality of life assessment in vascular disease: towards a consensus. Eur J Vasc Endovasc Surg 13:9-13

16. Korhonen SJ, Kantonen I, Pettila V et al (2003) Long-term survival and health-related quality of life of patients with ruptured abdominal aortic aneurysm. Eur J Vasc Endovasc Surg 25:350353

17. Bown MJ, Sutton AJ, Bell PR et al (2002) A meta-analysis of 50 years of ruptured abdominal aortic aneurysm repair. Br J Surg 89:714-730

18. Seiwert AJ, Elmore JR, Youkey JR et al (1995) Peter B. Samuels Award. Ruptured abdominal aortic aneurysm repair: the financial analysis. Am J Surg 170:91-96

19. Dick F, Diehm N, Galimanis A et al (2007) Surgical or endovascular revascularization in patients with critical limb ischemia: influence of diabetes mellitus on clinical outcome. J Vasc Surg 45:751-761

20. Kaplan EL, Meier P (1958) Nonparametric estimation from incomplete observations. J Am Stat Assoc 53:457-481

21. Bland JM, Altman DG (1997) Cronbach's alpha. BMJ 314:572

22. Sullivan M, Karlsson J, Ware JE (1994) SF-36 health questionnaire. Swedish manual and interpretation guide

23. Ware JE, Snow KK, Kosinski M (1993) SF-36 health survey manual and interpretation guide

24. Immer FF, Barmettler H, Berdat PA et al (2002) Effects of deep hypothermic circulatory arrest on outcome after resection of ascending aortic aneurysm. Ann Thorac Surg 74:422-425
25. Dubost C, Allary M, Oeconomos N (1952) Resection of an aneurysm of the abdominal aorta: reestablishment of the continuity by a preserved human arterial graft, with result after five months. Arch Surg 64:405-408

26. First Vascunet Database Report (2007) The European Society for Vascular Surgery. ISBN 1-903968-17-8

27. Magee TR, Scott DJ, Dunkley A et al (1992) Quality of life following surgery for abdominal aortic aneurysm. Br J Surg 79:1014-1016

28. Hennessy A, Barry MC, McGee H et al (1998) Quality of life following repair of ruptured and elective abdominal aortic aneurysms. Eur J Surg 164:673-677

29. Aquino RV, Jones MA, Zullo TG et al (2001) Quality of life assessment in patients undergoing endovascular or conventional AAA repair. J Endovasc Ther 8:521-528

30. Lloyd AJ, Boyle J, Bell PR et al (2000) Comparison of cognitive function and quality of life after endovascular or conventional aortic aneurysm repair. Br J Surg 87:443-447

31. Arko FR, Hill BB, Reeves TR et al (2003) Early and late functional outcome assessments following endovascular and open aneurysm repair. J Endovasc Ther 10:2-9

32. Malina M, Nilsson M, Brunkwall J et al (2000) Quality of life before and after endovascular and open repair of asymptomatic AAAs: a prospective study. J Endovasc Ther 7:372-379

33. Immer FF, Lippeck $\mathrm{C}$, Barmettler $\mathrm{H}$ et al (2004) Improvement of quality of life after surgery on the thoracic aorta: effect of antegrade cerebral perfusion and short duration of deep hypothermic circulatory arrest. Circulation 110:II250-II255

34. Crawford ES (1991) Ruptured abdominal aortic aneurysm. J Vasc Surg 13:348-350

35. Eckstein HH, Bruckner T, Heider P et al (2007) The relationship between volume and outcome following elective open repair of abdominal aortic aneurysms (AAA) in 131 German hospitals. Eur J Vasc Endovasc Surg 34:260-266

36. Mehta T, Venkata Subramaniam A, Chetter I et al (2006) Assessing the validity and responsiveness of disease-specific quality of life instruments in intermittent claudication. Eur J Vasc Endovasc Surg 31:46-52 